J. Clin. Chem. Clin. Biochem.

Vol. 16,1978 , pp. $425-428$

\title{
The Determination of Total Bilirubin with the Du Pont ACA with Respect to Neonatal Sera
}

\author{
By B. G. Blijenberg, H. J. Brouwer and B. Leijnse \\ Department of Clinical Chemistry, University Hospital, Rotterdam, The Netherlands
}

(Received December 5, 1977/March 6, 1978)

Summary: The interference of hemoglobin in the determination of total bilirubin with the Du Pont ACA was studied. The method seemed of limited value in our opinion especially with respect to neonatal sera. By changing the wavelength setting a rather broad plateau was found when the hemoglobin concentration was plotted against the actual bilirubin concentration. On the basis of these findings a new calibration graph was constructed and programmed into the ACA. The new method correlates well with two other micro bilirubin techniques.

\section{Die Bestimmung des Gesamtbilirubins mit dem Du Pont ACA unter Berücksichtigung von Serum Neugeborener}

Zusammenfassung: Die Störung durch Hämoglobin bei der Bestimmung des Gesamtbilirubins mit dem Du Pont ACA wurde untersucht. Die Methode ist unseres Erachtens besonders für die Bestimmung im Serum Neugeborener von begrenztem Wert. Durch Änderung des Wellenlängenbereichs wurde ein breiteres Plateau gefunden, wenn die Hämoglobinkonzentration gegen die aktuelle Bilirubinkonzentration graphisch aufgetragen wurde. Aufgrund dieser Ergebnisse wurde eine neue graphische Darstellung der Kalibrierung konstruiert und dem ACA eingegeben. Die neue Methode stimmt gut überein mit zwei anderen Mikromethoden für Bilirubin.

\section{Introduction}

The determination of bilirubin is very important in pediatrics. This statement is especially true for newborns (1). Bilirubin can be determined in different ways. Having a Du Pont Automatic Clinical Analyzer ${ }^{1}$ ) (ACA) in use in our laboratory, we found it worthwhile to study whether this instrument could meet the requirements for the determination of bilirubin in neonatal serum i.e.

a) sufficiently low sample volume

b) ease and speed of the analysis and

c) reliability of the result.

It is known from the Du Pont literature that the ACA procedure for total bilirubin is subject to interference by hemoglobin. In general, the interference of hemoglobin with the determination of bilirubin is well known in clinical chemistry. Especially in the Scandinavian literature, much attention has been paid to this problem $(2,3)$. All bilinubin methods in use in the clinical chemistry laboratory are more or less subject to a disturbance by hemoglobin.

1) E.I. du Pont de Nemours and Co (Inc.), Instrument Products Division, Wilmington, Del. 19.89 .8 U.S.A.
The determination of bilirubin in the diagnosis and the treatment of hyperbilirubinemia in newborns puts a twofold burden on the laboratory. It is known that the sera of newborns contain a higher concentration of hemoglobin than normally seen in adults (heel puncture!). Michaelsson \& Sjölin (3) reported a study in which $86 \%$ of sera had a hemoglobin content of more than $60 \mu \mathrm{mol} / 1$ (Fe). Furthermore the therapeutic measures may be more drastic than necessary as a consequence of such results. Therefore it seemed worthwhile to start the study with the measurement of the interference of hemoglobin in the bilirubin method (TBIL = total bilirubin) of the Du Pont ACA.

\section{Materials and Methods}

Bilirubin

Standard bilirubin preparations were prepared according to the Recommendations of the well known Joint Committee (4),

Hemoglobin and derivatives

Serum samples were artificially enriched with hemoglobin by the addition of small volumes of red cell hemolysate, as well as a hemoglobin solution prepared according to Van Assendelft (5). No difference between these preparations was detected. The hemoglobin derivatives were prepared according to Van Assendelft. 
Automatic Clinical Analyzer (ACA)

The Du Pont ACA is an analyzing system in which all "chemical" operations are automated except the introduction of the sample. A sample cup is filled with serum and brought into the system together with the reagents. The reagents for each test are packaged in a special pack which serves both as the reaction chamber and test cuvette for photometric analysis. The total reaction time is 7 minutes.

\section{Results}

We used bilirubin standards (see Materials and Methods) to which hemoglobin (see Materials and Methods) was added. The results are given in table 1.

The consequences of the results shown in table 1 are, in our opinion, rather embarassing. There is no doubt that the method is of limited value in pediatric chemistry, and it is of doubtful value for hemolyzed adult sera. The results shown in table 1 suggest a combined interference: a spectrometric interference and an interaction in the diazo reaction. This suggestion is based on the rise of the blank result (see column : blank) with increasing hemoglobin concentrations and the non-linear fall of the standard sera. We found it worthwhile and simple to deal with the spectrometric part of the disturbance by changing the hemoglobin molecule and choosing a different filter combination. We tried deoxygenated hemoglobin and hemiglobin. However, the results were comparable to those mentioned in table 1 . As can be seen in figure 1 the combination 510-600 $\mathrm{nm}$ seems better than the combination 540-600 $\mathrm{nm}$. Indeed we found a higher response, but, of course, the hemoglobin still interfered.

Tab. 1. Influence of hemoglobin on several bilirubin standard sera determined with the original ACA procedure.

\begin{tabular}{rllll}
\hline $\begin{array}{l}\text { Hemoglobin } \\
(\mu \mathrm{mol} / \mathrm{l})\end{array}$ & \multicolumn{4}{c}{ Bilirubin $(\mu \mathrm{mol} / \mathrm{l})$} \\
& Blank & Standard 1 & Standard 2 & Standard 3 \\
\hline 0 & 0 & 83 & 167 & 250 \\
67 & 2 & 49 & 121 & 185 \\
133 & 5 & 39 & 97 & 161 \\
267 & 12 & 31 & 86 & 136 \\
\hline
\end{tabular}

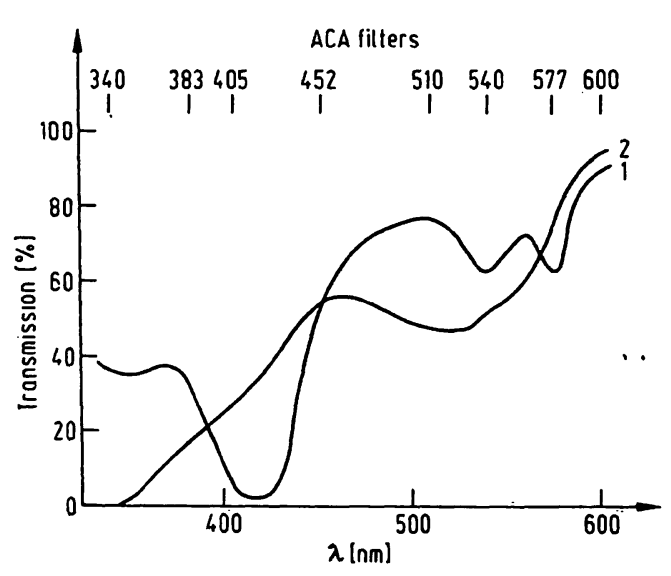

Fig. 1. Absorption spectrum of hemoglobin (1) and a bilirubin standard serum processed in the ACA (2).
However, in looking at the influence of various hemoglobin concentrations we found a surprising effect. As can be seen in figure 2 a rather broad plateau is reached with higher hemoglobin concentrations.

So, by introducing hemoglobin in some way in the "TBIL pack" it should be possible to construct a derived calibration graph. This can easily be done by adding hemoglobin to the sixth diluent can on the ACA, because this can is not in use.

To know how much hemoglobin would be needed it was necessary to know the concentration in the samples taken from babies. In a careful study Michaëlsson has already given the data obtained with various puncturing techniques (3). In our laboratory we used the Crosby \& Furth hemoglobin determination on 129 samples. The average value was $106 \mu \mathrm{mol} / \mathrm{l}(\mathrm{Fe})$ with a lowest value of $5 \mu \mathrm{mol} / 1$ (Fe) and a highest of $449 \mu \mathrm{mol} / \mathrm{l}(\mathrm{Fe})$, i.e. comparable to the results of Michaëlsson et al. On the basis of these data we decided to add a quantity of hemoglobin to the can that corresponded to a serum level of about $150 \mu \mathrm{mol} / 1$ (Fe). This meant the addition of $1 \mathrm{ml}$ hemoglobin solution (5), concentration $5 \mathrm{mmol}$ $\mathrm{Hb}(\mathrm{Fe})$ per liter, to 31 determineralized water. This addition is based on a sample volume of $60 \mu \mathrm{l}$.

To check if the foregoing results were practical we measured several standards to construct a calibration graph. The standard sera also contained $100 \mu \mathrm{mol}$ hemoglobin/l $(\mathrm{Fe})$. The calibration graph was found to be linear up to $340 \mu \mathrm{mol} / 1$. We changed the decoding system and letter wheel in the printer (now "BBIL" was printed in stead of "TBIL") in order to avoid mistakes. This can be done easily by wiping off the first black bar on the "TBIL pack" header and by relacing the photometer control board in the computer system. So, with the same chemistry the method looked like a new method for the instrument.

The changes we performed were checked by studying the hemoglobin interference with the new measuring

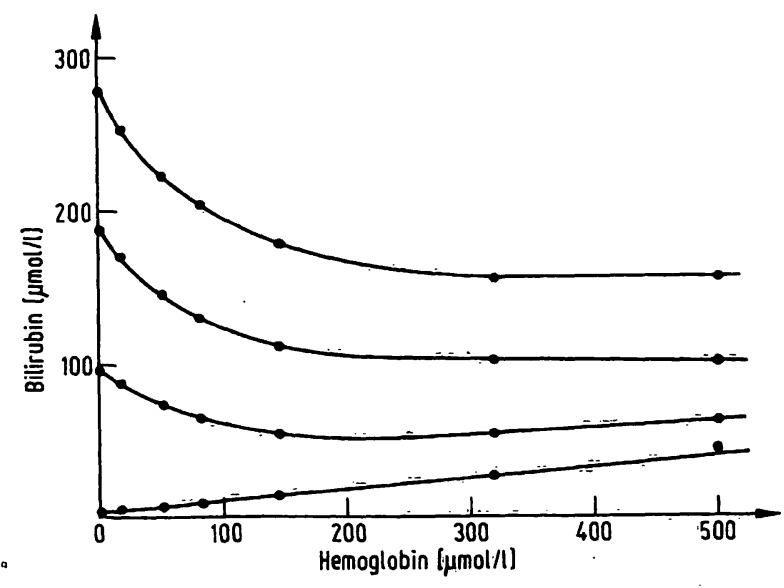

Fig. 2. The interference of hemoglobin' with the "TBIL" determination using the filter combination $51 \overline{0}-600 \mathrm{~nm}$ 


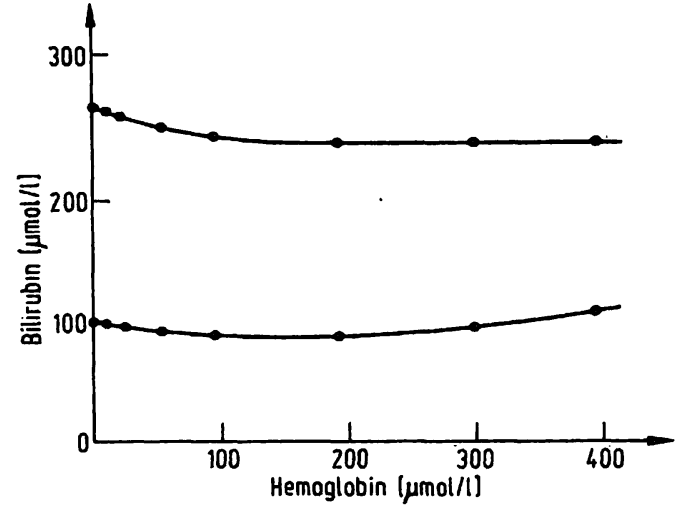

Fig. 3. The interference of hemoglobin with the revised ACA procedure (= "BBIL").

system and by starting two correlation studies. In figure 3 the hemoglobin interference of the "BBIL" method is shown.

The first correlation was done with an instrument based on the direct measurement of serum at two wavelengths (455 and $575 \mathrm{~nm}$ ), called the OHC Photo Ictometer (6). The samples were measured at random. The results are shown in figure 4. Assuming a linear regression between the two variables the equation of the regression line was found to be

$$
\mathrm{y}=0.97 \mathrm{x}+4 \quad \mathrm{n}=155
$$

with a correlation coefficient of 0.87 . The average value of the results obtained with the Ictometer $(x)$ was found to be $152 \mu \mathrm{mol} / 1$ while the ACA value (y) was $156 \mu \mathrm{mol} / 1$.

The influence of hemoglobin on the Ictometer can be shown in figure 5 in which "elevated" samples (hemo-

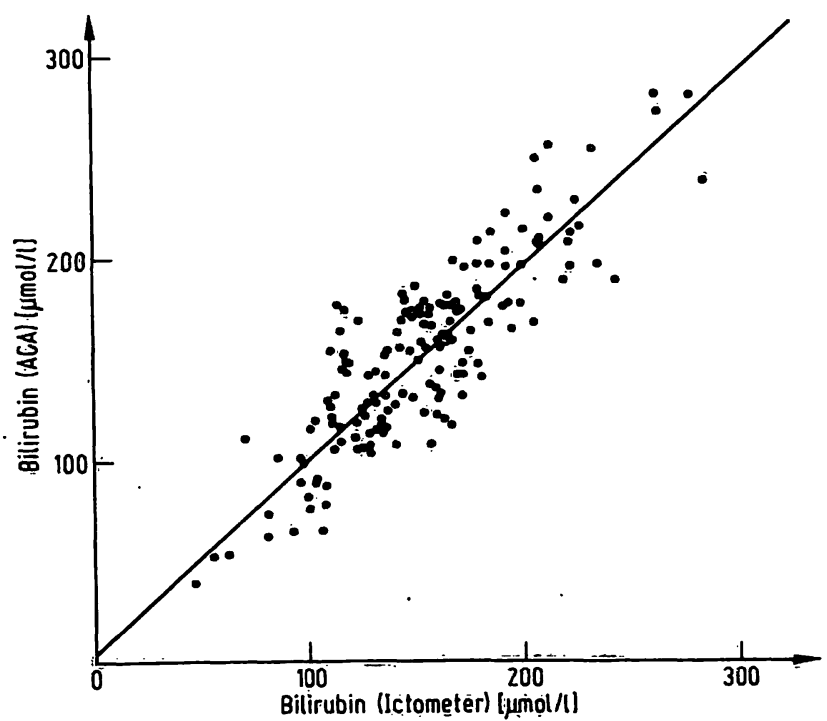

Fig. 4. Split-sample comparison graph between the "BBIL" and the Ictometer.

Regression equation ( $y$ on $x$ ) : $y=0.97 x+4, r=0.87, n=155$. globin concentration $>150 \mu \mathrm{mol} / \mathrm{l}$ ) are plotted and in figure 6 in which the influence is measured.

Our second correlation was a comparison with the method of Hertz et al which is also very suitable for the determination of bilirubin in neonatal sera (7). The influence of hemoglobin in this method is less than measured with the Ictometer, as can be seen in Figure 6 The results of this comparison is shown in figure 7. The equation of the regression line was

$$
y=1.09 x+14 \quad n=160
$$

with a correlation coefficient of 0.90 . Here we found an average value of $138 \mu \mathrm{mol} / 1$ for $\mathrm{x}(=$ Hertz $)$ and 164 $\mu \mathrm{mol} / 1$ for $\mathrm{y}(=\mathrm{ACA})$.

Finally we performed precision studies. The "withinrun" and "day-to-day" estimates of precision of two standard samples (see Materials and Methods) are shown in table 2.

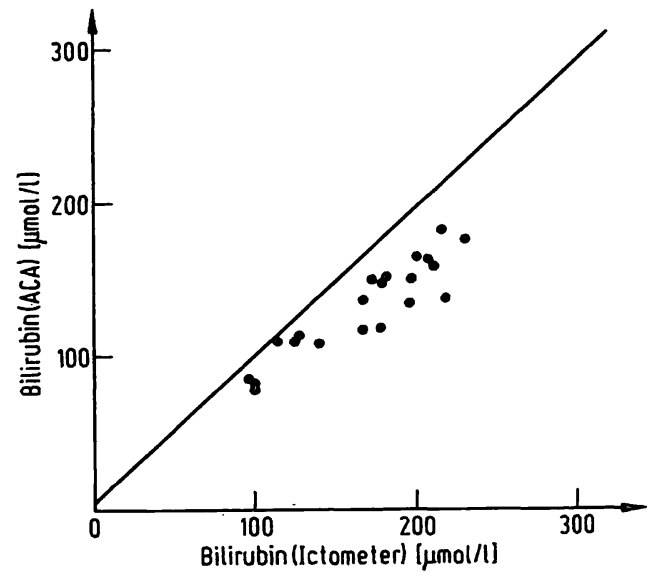

Fig. 5. Split-sample comparison graph between the "BBIL" and the Ictometer for samples with a hemoglobin concentration $>150 \mu \mathrm{mol} / 1$.

The straight line represents the regression line mentioned in figure 4.

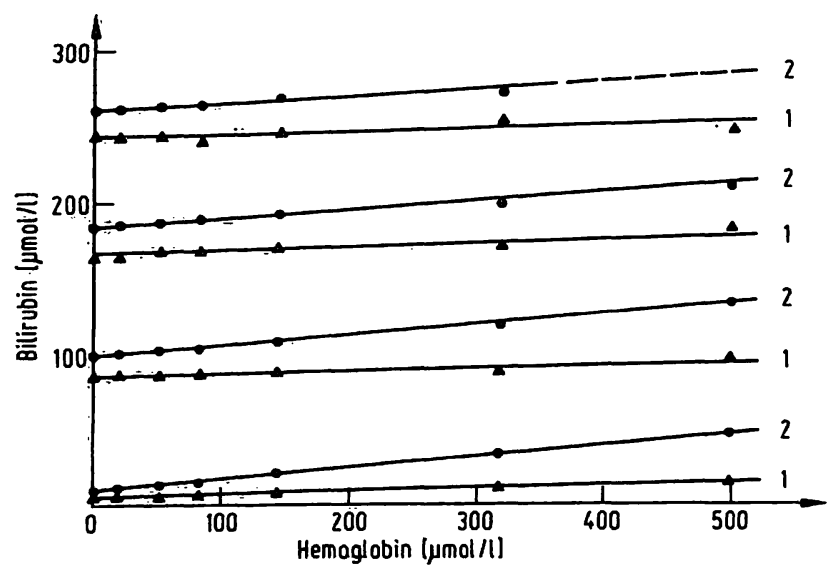

Fig. 6. The interference of hemoglobin with the Hertz' technique (1) and the Ictometer (2). 


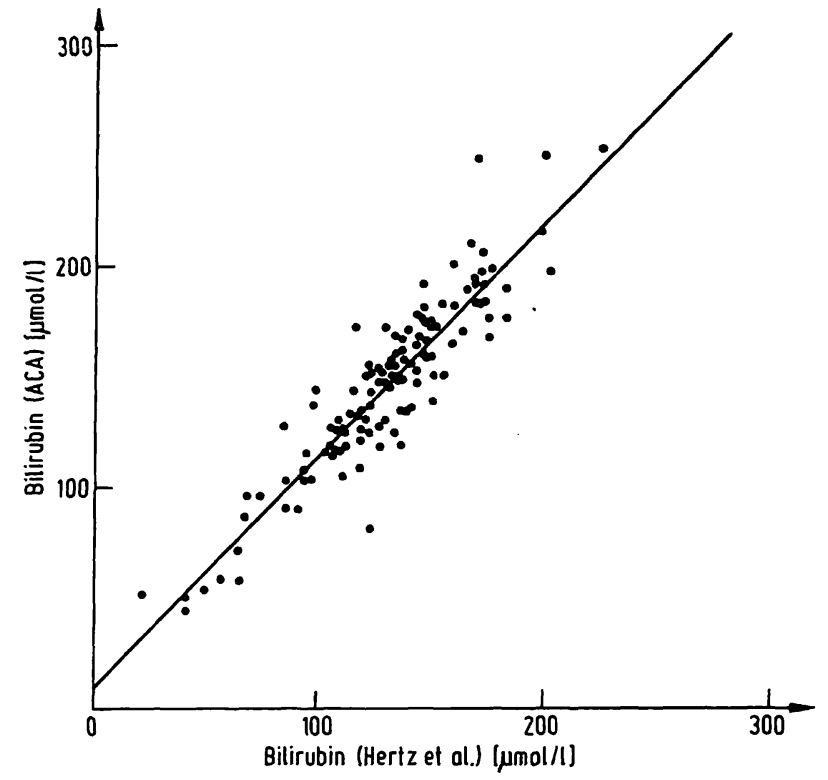

Fig. 7. Split-sample comparison graph between the "BBIL" and the Hertz' technique.

Regression equation ( $\mathrm{y}$ on $\mathrm{x}$ ) : $y=1.09 x+14, r=0.90, n=160$.

Tab. 2. Precision data.

\begin{tabular}{lllll}
\hline 1. Within-run "BBIL" & Lot 1, & $\mathrm{n}=20$ & Lot 2, & $\mathrm{n}=12$ \\
$\overline{\mathrm{x}}(\mu \mathrm{mol} / \mathrm{l})$ & 78 & 263 & 77 & 232 \\
CV (\%) & 5.2 & 2.9 & 4.9 & 3.2 \\
2. Day-to-day "BBIL" & Lot 1, & $\mathrm{n}=27$ & Lot 2, & $\mathrm{n}=20$ \\
$\overline{\mathrm{X}}(\mu \mathrm{mol} / \mathrm{l})$ & 79 & 242 & 77 & 246 \\
CV (\%) & 6.1 & 6.0 & 7.2 & 3.7 \\
3. Day-to-day Hertz & Lot 1, & $\mathrm{n}=26$ & Lot 2, & $\mathrm{n}=28$ \\
$\overline{\mathrm{X}}(\mu \mathrm{mol} / \mathrm{l})$ & 81 & 238 & 82 & 246 \\
CV (\%) & 6.4 & 2.7 & 4.8 & 4.1
\end{tabular}

Lot 1 and 2 "BBIL" $=2$ different lot numbers for the test packs, 2 different standard sera and 2 cans diluent Lot 1 and 2 "Hertz" $=2$ different standard sera.

\section{Discussion}

In the Introduction we mentioned three requirements which must be satisfied in a determination of bilirubin in neonatal serum. The ACA can easily satisfy requirements $a$ and $b$. Requirement $c$ concerns the criteria for the reliability of a result. In fact, this devolves on the clinical usefulness of the method.

With respect to the determination of bilirubin in the serum of newborns, one can roughly separate the results into two parts i.e. those lying under $300 \mu \mathrm{mol} / 1$ and those higher than $300 \mu \mathrm{mol} / 1$. The results higher than $300 \mu \mathrm{mol} / \mathrm{l}$ can be near or at the decision level in the case of an exchange transfusion of ery throblastotic newborns. Therefore a physician can ask for maximal precision at this level, while the results lower than $300 \mu \mathrm{mol} / 1$ can be permitted a lower precision. In his article on the medical significance of laboratory results Barnett mentions a coefficient of variation at the decision level of $7.5 \%$ (8). This figure is in agreement with the management of the pediatric department of our hospital. Thus, from table 2 , the conclusion can be drawn that, with the changes described in this article, the ACA can be used for the determination of total bilirubin in neonatal sera. In addition, the correlation with other methods is acceptable. There is, in our opinion, no point in performing extensive statistical calculations to prove the pragmatic conclusion just mentioned, because in all methods used a systematic error, though sometimes low, still exists. However, the problem of accuracy still exists. Serum samples with a very low or with a very high hemoglobin content give values that are too high, as can be seen from figure 3. It is very difficult to evaluate these "outliers". In our study the number of samples with a hemoglobin content between 20 and $400 \mu \mathrm{mol} / 1(\mathrm{Fe})$ was 95 per cent. Because of the problem of accuracy we have decided to continue the study. In the meantime, it seemed worthwhile to report the solution of the interference problem, because it works satisfactorily according to the opinion of the physicians involved in our hospital. We have already estimated more than a thousand samples in this way.

\section{Acknowledgements}

We thank the Du Pont Co. for providing the test packs. Furthermore, thanks are due to Ir. N.C. den Boer, Sophia Childrens Hospital, and Mrs. L.J. Verhoog-Perret and Mr. W. Kafoe.

\section{References}

1. Young, D. S. \& Hicks, J. M. (1976), The Neonate, John Wiley, New York.

2. Michaëlsson, M., Nosslin, B. \& Sjölin, S. (1965), Pediatrics, 35, 925-931.

3. Michaëlsson, M. \& Sjölin, S. (1965), Acta Paediatr. Sçand., $54,325-330$.

4. Recommendation on a uniform bilirubin standard (1962), Clin. Chem. 8, 405-407.
5. Van Assendelft, O. W. (1970), Spectrophotometry of haemoglobin derivatives, Van Gorcum, Assen.

6. Stein, S. M., McKinley, I., Horn, D. B. \& Keay, A. J. (1974), Clin. Chim. Acta, $54,107-113$.

7. Hertz, H., Dybkaer, R. \& Lauritzen, M. (1974), Scand. J. Clin. Lab. Invest. 33, 215-230.

8. Barnett, R. N. (1968), Am. J. Clin. Pathol., 50, 671-676.

9. Crosby, W. H. \& Furth, F. W. (1960), Blood, 1l, 380.

Dr. B. G. Blijenberg

Acad. Ziekenhuis Rotterdạm-Dijkzigt Dr. Molewaterplein 40 Rotterdam-3002 